\title{
Recurrent Spontaneous Abortion of Immune Origin and HLA Sensitization Immunotherapy
}

\author{
Isabela Mansur Bandeira', Juliana Vasconcelos' ${ }^{1}$, Thiago Luiz Bandeira ${ }^{1}$, \\ Jorge Oliveira1, Carla Goulart ${ }^{1}$, Aderbal Sabra², Luiz Werber-Bandeira1
}

\begin{abstract}
${ }^{1}$ Clinical and Experimental Immunology and Allergy Service, Santa Casa da Misericordia do Rio de Janeiro, Rio de Janeiro, Brazil ${ }^{2}$ Food Allergy and Autism Unit of the Clinical Immunology Service, Santa Casa da Misericordia do Rio de Janeiro, Rio de Janeiro, Brazil
\end{abstract}

Email: werberbandeira@imunoderm.com.br

How to cite this paper: Bandeira, I.M., Vasconcelos, J., Bandeira, T.L., Oliveira, J., Goulart, C., Sabra, A. and Werber-Bandeira, L. (2022) Recurrent Spontaneous Abortion of Immune Origin and HLA Sensitization Immunotherapy. Advances in Reproductive Sciences, 10, 12-18.

https://doi.org/10.4236/arsci.2022.101002

Received: October 19, 2021

Accepted: February 19, 2022

Published: February 22, 2022

Copyright (c) 2022 by author(s) and Scientific Research Publishing Inc. This work is licensed under the Creative Commons Attribution International License (CC BY 4.0).

http://creativecommons.org/licenses/by/4.0/

\begin{abstract}
Introduction: Spontaneous abortion is defined as pregnancy loss before the twentieth week and Recurrent Spontaneous Abortion (RSA) is defined as at least three spontaneous and successive pregnancy losses in the same period. Among the different types of immunological causes, this study refers specifically to the alloimmune cause. Women with RSA of alloimmune cause share a greater number of Human Leukocyte Antigen (HLA) with their husbands, leading to the inhibition of the production of anti-paternal asymmetric blocking antibodies, which would protect embryonic cells. Objectives: To evaluate the effect of immunotherapy with paternal HLA-sensitizing mononuclear cells in cases of RSA through the positivity of the cross-match test and its efficacy in pregnancy success. Patients and Methods: Written consent was obtained, 12 couples with a history of RSA presenting negative cross-match were included in the study. Blood samples were collected from the couple for cross-matching and the separation of paternal mononuclear cells. Immunotherapy was performed with paternal mononuclear cells intradermally in the wives' arms on day (D) 0 , D15 and D30. After the third dose, a second evaluation of the crossmatch tests was performed. Results and Discussion: The age of the wives ranged from 28 to 41 years, with a mean of 34.5 years. The twelve couples had a negative cross-match test (100\%). Eleven couples (96.6\%) tested positive in the crossmatch test after immunotherapy. Of these, 10 (90.9\%) had successful pregnancies. Immunotherapy with paternal mononuclear cells showed an excellent ability to sensitize the maternal immune system, with positive cross-match, resulting in a successful pregnancy.
\end{abstract}

\section{Keywords}

Spontaneous Abortion, Immunotherapy, Pregnancy, Maternal-Fetal 
Relationships

\section{Introduction}

Pregnancy is a challenge to the maternal immune system because the mother must tolerate the paternal alloantigens present in the fetus, which represents a paradoxical tolerance to a semiallogeneic transplant [1] [2]. The immune relationship between the mother and the fetus is a bidirectional communication, determined on the one hand, by the presentation of fetal antigens to the mother and, on the other hand, by the recognition of and reaction to these antigens by the maternal immune system. The recognition (by the mother) of paternal Human Leukocyte Antigens (HLA) exposed by the fetus, specific HLA in the placenta, cytokines produced by Th2 lymphocytes present in the placenta and produced by the fetus, special cytotoxicity mechanisms, and asymmetric antibody factors could explain the protective mechanisms of the fetus [3] [4].

The inadequate recognition of fetal antigens and the increased similarity of HLA between the mother and the father could lead to the loss of defense mechanisms and, consequently, to Recurrent Spontaneous Abortion (RSA) [5]. As an example, the protective role of HLA-G, restrictively expressed in trophoblasts has been studied and considered in the literature [6]. The interaction of this molecule with the receptors of cells from the maternally immune system in the uterus can contribute to successful pregnancies acting as a protective factor. In the past 20 years, studies associated with HLA-G have been continually published [7].

From the immunological point of view, during the peri-implantation period, the Th1 pro-inflammatory immune response is predominant and its benefits invading trophoblasts. After placental implantation, there is a shift between Th1 and Th2 cytokines [8], and the level of Th1 cytokines (IL-2, IFN-g, TNF-a, and IL-6) reduces, while the level of Th2 cytokines (IL-4, IL-10), the Th2 anti-inflammatory immune response increases in that area protecting and allowing the fetal and placental development.

Women with alloimmune cause of Recurrent Spontaneous Abortion (RSA), as two or more miscarriages before 20 weeks of gestation, tend to share a greater number of Human Leukocytes Antigens (HLA) with husbands which could inhibit the mother from making Anti-Paternal Cytotoxic Antibody (APCA), Mixed Lymphocyte Reaction Blocking Antibody (MLR-Bf), and asymmetric IgG protecting the fetus [9].

\section{Immunotherapy with paternal mononuclear cells}

As proposed by Taylor and Faulk in 1981 [10] and by Harris and Pierangeli, 1998 [11], immunotherapy with Paternal Mononuclear Cells (PLI) induces a humoral response with the production of anti-idiotype and asymmetric-blocking antibodies. It has been shown that in cases of poor prognosis, this procedure is ef- 
fective in allowing the pregnant woman with immune abortion to return to the reactional state similar to the physiological [12]. The production of asymmetric antibodies masks paternal HLA, one of the fetal antigens, preventing maternal rejection of the fetus with cytotoxic T lymphocytes [13] [14] [15] [16] [17] [18]. According to Wu et al. 2014, immunotherapy may induce a decrease in the Th17/Treg ratio and the Treg bias, which may be beneficial to the maintenance of pregnancy regulating the levels of the asymmetric antibodies, unable to activate immune effector mechanisms [19]. The induction of the HLA response among couples after mononuclear therapy is the criterion for immunization. Usually, in pregnant women, anti-idiotype and asymmetric-blocking antibodies are present in high levels, which are beneficial to pregnancy maintenance, but in women with RSA, these levels are significantly lower.

Concerning the analysis of the possible side effects of PLI, in a follow-up study performed in Germany and covering a high number of women in a period of seven years, Kling et al. 2006 reported that there were no cases of anaphylaxis, autoimmune, or graft versus host diseases [20].

We understand that the contribution of this study to the state of the art of knowledge on RSA is the positive result of immunotherapy with lymphocytes. The use of fresh leukocytes probably enabled this very effective result when compared to the results in the literature with stored and frozen leukocytes. It is possible that the effectiveness of immunotherapy can be reduced with this practice.

\section{Design and Objectives}

This is a retrospective cohort study with longitudinal follow-up, with the objective of evaluating the effect of immunotherapy with paternal HLA-sensitizing mononuclear cells in cases of RSA through the positivity of the cross-match test and the efficacy of this therapy based on pregnancy success.

\section{Patients and Methods}

After signing an informed consent form, patients from the Immunology and Clinical and Experimental Allergy Service of Santa Casa da Misericórdia do Rio de Janeiro answered a standard questionnaire to assess eligibility for the study [21]. The couples who met the following inclusion criteria were considered eligible: diagnosis of RSA of undetermined cause with possibility of immune cause, any ethnicity, and wife aged 18 years or older and negative cross-match between the couple.

Twelve couples $(\mathrm{n}=12)$ were enrolled in the study over a six-year period from 2008 to 2014. Peripheral blood samples were collected from the couples for crossmatching tests and for obtaining Peripheral Blood Mononuclear Cells (PBMC) to be used for immunotherapy. The PBMC isolation was carried out by the Ficoll-Hypaque gradient technique.

Immunotherapy was performed by using an average of 60 to $500 \times 10^{6}$ fresh mononuclear cells intradermally with 15-day intervals in three sessions Day (D) 
0, D15 and D30. After the third dose, a second cross-match test between the couple was performed.

\section{Results}

We analyzed twelve couples with recurrent spontaneous abortion of immune cause who were diagnosed by the absence of any other causes and by the negative crossmatch between wife and husband. The age of the wives ranged from 28 to 41 years old, with a mean of 34.5 years. After the third dose of immunotherapy with the husband's mononuclear cells, the second evaluation of the cross-matching was performed. Eleven of the 12 participating couples (96.6\%) tested positive in the cross-match test after immunotherapy. Of these, 10 (90.9\%) had a successful pregnancy, and one didn't get pregnant. It is important to note that pregnancy can be attempted for up to 12 months after immunotherapy with positive cross-match.

\section{Discussion}

Evaluation of the efficacy of HLA sensitization immunotherapy using mononuclear cells from the spouses has been studied worldwide. In 2002, Makino [22], evaluated the efficacy of the procedure by comparing the results of immunotherapy in a group of women with RSA who adhered against a group that did not spontaneously adhere to treatment. In that study, in a case-control design, there was a significant difference in treatment success characterized by non-interruption of pregnancy. In our study, the success of immunotherapy was measured in terms of occurrence, by assessing the percentage of pregnancy success in the treated group.

Usually, the assessment of the immunotherapy methods is based on evidence by comparing trials and using comparison to no treatment; but this approach does not specifically elucidate which changes at the immunological level are actually taking place. The lack of gold standard laboratory criteria by which immunemediated mechanisms can be identified hampers the achievement of this knowledge. Additionally, the scenario still has confounding factors and side effects that should also be considered for a precise evaluation of PLI.

The process of successful pregnancy goes through a paradoxical state that contradicts the basic foundations of immunology where the immune system has the primary function of genetic surveillance considering the fetus an allograft; however, rejection does not occur. Asymmetric-blocking antibodies, which favor pregnancy, have a special molecular configuration and do not do what would be expected, i.e., cell death induction through complement-dependent cell cytotoxicity. These antibodies are increased during pregnancy and should remain so until the end of pregnancy.

The comparison between immunotherapy performed with mononuclear cells from the father and with autologous mononuclear cells was tested by several research groups. Data from the literature report $67 \%-86 \%$ of success compared to $36 \%$ of autologous and third-party mononuclear cell therapy $(p<0.05)$ [23] [24].

The negative cross-match would represent negative micro lymphocytoxicity; 
consequently, the immune system of the wives would not react to the husbands, not protecting the fetus. In our study, immunotherapy with paternal mononuclear cells showed excellent results, demonstrating the capacity of sensitization of the maternal immune system as shown by the high percentage of positivity of the crossmatch test. In addition, immunotherapy showed excellent efficacy, resulting in pregnancy success.

\section{Conclusion}

The success of the immunotherapy obtained in this study, corroborates data from international trials, suggesting that the mechanisms of pregnancy and the use of immunotherapy should be discussed in greater depth, as well as supporting the more frequent use of this HLA sensitization therapy in cases of recurrent spontaneous abortion of immune cause.

\section{Acknowledgements}

We thank the Health and Sustainability Association - ASAS.

\section{Funding}

This study was financial supported by ImunoDerm Clinics.

\section{Conflicts of Interest}

The authors declare no conflicts of interest regarding the publication of this paper.

\section{References}

[1] Wegmann, T.G. (1980) Why Didn't Your Mother Reject You? Canadian Medical Association Journal, 22, 991-993.

[2] Innes, A., Stewart, G.M., Thomso, M.A., Cunningham, C. and Catto, G.R. (1988) Human Placenta-An Antibody Sponge? American Journal of Reproductive Immunology and Microbiology, 17, 57-60. https://doi.org/10.1111/j.1600-0897.1988.tb00203.x

[3] Avril, T., Jarousseau, A.C., Watier, H., Boucraut, J., Le Bouteiller, P., et al. (1999) Trophoblast Cell Line Resistance to NK lysis Mainly Involves an HLA Class I-Independent Mechanism. The Journal of Immunology, 162, 5902-5909. http://www.jimmunol.org/content/162/10/5902

[4] Zenclussen, A.C., Fes, S., Bussse, P., Joachim, R., Klapp, B.F. and Arcck, P.C. (2002) Questioning the Th1/Th2 Paradigm in Reproduction: Peripheral Levels of IL-12 Are Down-Regulated in Miscarriage Patients. American Journal of Reproductive Immunology, 48, 245-251. https://doi.org/10.1034/j.1600-0897.2002.01136.x

[5] Dahl, M., Vauvert, T.F.T. and Hviid, T.V. (2011) Human Leukocyte Antigen Class Ib Molecules in Pregnancy Success and Early Pregnancy Loss. Human Reproduction, 18, 92-109. https://doi.org/10.1093/humupd/dmr043

[6] Xu, X., Zhou, Y. and Wei, H. (2020) Roles of HLA-G in the Maternal-Fetal Immune Microenvironment. Fronts in Immunology, 11, Article No. 592010. https://doi.org/10.3389/fimmu.2020.592010

[7] Zhuang, B., Shang, J. and Yao, Y. (2021) HLA-G: An Important Mediator of Maternal- 
Fetal Immune-Tolerance. Fronts in Immunology, 12, Article No. 744324. https://doi.org/10.3389/fimmu.2021.744324

[8] Yokoo, T., Takakuwa, K., Ooki, I., Kikuchi, A., Tamura, M. and Tanaka, K. (2006) Alteration of TH1 and TH2 Cells by Intracellular Cytokine Detection in Patients with Unexplained Recurrent Abortion before and after Immunotherapy with the Husband's Mononuclear Cells. Fertility and Sterility, 85, 1452-1458.

https://doi.org/10.1016/j.fertnstert.2005.10.058

[9] Wang, W., Sung, N., Gilman-Sachs, A. and Kwak-Kim, J. (2020) T Helper (Th) Cell Profiles in Pregnancy and Recurrent Pregnancy Losses: Th1/Th2/Th9/Th17/Th22/Tfh Cells. Fronts in Immunology, 11, 20-25. https://doi.org/10.3389/fimmu.2020.02025

[10] Taylor, C. and Faulk, W.P. (1981) Prevention of Recurrent Abortion with Leukocyte Transfusions. The Lancet, 318, 68-70. https://doi.org/10.1016/S0140-6736(81)90413-X

[11] Harris, E.N. and Pierangeli, S.S. (1998) Utilization of Intravenous Immunoglobulin Therapy to Treat Recurrent Pregnancy Loss in the Antiphospholipids Syndrome: A Review. Scandinavian Journal of Rheumatology, 27, 97-102. https://doi.org/10.1080/03009742.1998.11720777

[12] Carp, H. (2019) Immunotherapy for Recurrent Pregnancy Loss. Best Practice \& Research Clinical Obstetrics \& Gynaecology, 60, 77-86. https://doi.org/10.1016/j.bpobgyn.2019.07.005

[13] MacIntyre, J.A. and Faulk, W.P. (1986) Clinical Value of Research in Chronic Spontaneous Abortion. American Journal of Reproductive Immunology and Microbiology, 10, 121-126.

[14] Cauchi, M.N., Lim, D., Young, D.E., Kloss, M. and Pepperell, R.J. (1991) Treatment of Recurrent Aborters by Immunization with Paternal Cells-Controlled Trial. American Journal of Reproductive Immunology, 25, 16-17. https://doi.org/10.1111/j.1600-0897.1991.tb01057.x

[15] Illeni, M.T., Marelli, G., Parazzini, F., Acaia, B., Bocciolone, L., et al. (1994) Immunotherapy and Recurrent Abortion: A Randomized Clinical Trial. Human Reproduction, 9, 1247-1249. https://doi.org/10.1093/oxfordjournals.humrep.a138687

[16] Ito, K., Tanaka, T., Tsutsumi, N., Obata, F. and Kashiwagi, N. (1999) Possible Mechanism of Immunotherapy for Maintaining Pregnancy in Recurrent Spontaneous Aborters: Analysis of Anti-Idiotypic Antibodies Direct against Autologous T-Cell Receptors. Human Reproduction, 14, 650-655. https://doi.org/10.1093/humrep/14.3.650

[17] Ramhorst, R., Agriello, E., Zitterman, S., Pando, M., Larriba, J., et al. (2000) Is the Paternal Mononuclear Cells' Immunization a Successful Treatment for Recurrent Spontaneous Abortion? American Journal of Reproductive Immunology, 44, 129-135. https://doi.org/10.1111/j.8755-8920.2000.440301.x

[18] Pandey, M.K., Saxena, V. and Agrawal, S. (2003) Characterization of Mixed Lymphocyte Reaction Blocking Antibodies (MLR-Bf) in Human Pregnancy. BMC Pregnancy and Childbirth, 3, Article No. 2. https://doi.org/10.1186/1471-2393-3-2

[19] Wu, L., Luo, L.H., Zhang, Y.X., Li, Q., Xu, B., Zhou, G.X., Luan, H.B. and Liu, Y.S. (2014) Alteration of Th17 and Treg Cells in Patients with Unexplained Recurrent Spontaneous Abortion before and after Lymphocyte Immunization Therapy. Reproductive Biology and Endocrinology, 12, Article No. 74. https://doi.org/10.1186/1477-7827-12-74

[20] Kling, C., Steinmann, J., Westphal, E., Magez, J. and Kabelitz, D. (2006) Adverse Effects of Intradermal Allogeneic Lymphocyte Immunotherapy: Acute Reactions and Role of Autoimmunity. Human Reproduction, 21, 429-435. 
https://doi.org/10.1093/humrep/dei316

[21] Clifford, K., Rai, R., Watson, H. and Regan, I. (1994) An Informative Protocol for the Investigation of Recurrent Miscarriage: Preliminary Experience of 500 Consecutive Cases. Human Reproduction, 9, 1328-1332.

[22] Makino, T. (2002) Recurrent Reproductive Wastage and Immunologic Factors. American Journal of Reproductive Immunology, 48, 266-268.

[23] Daya, S. and Gunby, J. (1994) The Effectiveness of Allogenic Leukocyte Immunization in Unexplained Primary Recurrent Spontaneous Abortion. American Journal of Reproductive Immunology, 32, 294-302.

[24] Carp, H.J.A., Toder, V. and Torchinsky, A. (1997) Allogenic Leukocyte Immunization after Five or More Miscarriages. Recurrent Miscarriage Immunotherapy Trialists Group. Human Reproduction, 12, 250-255. 